\title{
Depression as an independent predictor of postoperative delirium in spine deformity patients undergoing elective spine surgery
}

\author{
Aladine A. Elsamadicy, BE, ${ }^{1}$ Owoicho Adogwa, MD, MPH, ${ }^{2}$ Emily Lydon, BS, ${ }^{1}$ \\ Amanda Sergesketter, BS, Rayan Kaakati, BS, ${ }^{1}$ Ankit I. Mehta, MD, ${ }^{3}$ Raul A. Vasquez, MD, ${ }^{4}$ \\ Joseph Cheng, MD, MS, ${ }^{5}$ Carlos A. Bagley, MD, ${ }^{6}$ and Isaac O. Karikari, MD' \\ ${ }^{1}$ Department of Neurosurgery, Duke University Medical Center, Durham, North Carolina; ${ }^{2}$ Department of Neurosurgery, Rush \\ University Medical Center, Chicago; ${ }^{3}$ Department of Neurosurgery, The University of Illinois at Chicago, Illinois; ${ }^{4}$ Department \\ of Neurosurgery, University of Kentucky, Lexington, Kentucky; ${ }^{5}$ Department of Neurosurgery, Yale University, New Haven, \\ Connecticut; and ${ }^{6}$ Department of Neurosurgery, University of Texas South Western, Dallas, Texas
}

OBJECTIVE Depression is the most prevalent affective disorder in the US, and patients with spinal deformity are at increased risk. Postoperative delirium has been associated with inferior surgical outcomes, including morbidity and mortality. The relationship between depression and postoperative delirium in patients undergoing spine surgery is relatively unknown. The aim of this study was to determine if depression is an independent risk factor for the development of postoperative delirium in patients undergoing decompression and fusion for deformity.

METHODS The medical records of 923 adult patients (age $\geq 18$ years) undergoing elective spine surgery at a single major academic institution from 2005 through 2015 were reviewed. Of these patients, 255 (27.6\%) patients had been diagnosed with depression by a board-certified psychiatrist and constituted the Depression group; the remaining 668 patients constituted the No-Depression group. Patient demographics, comorbidities, and intra- and postoperative complication rates were collected for each patient and compared between groups. The primary outcome investigated in this study was rate of postoperative delirium, according to DSM-V criteria, during initial hospital stay after surgery. The association between depression and postoperative delirium rate was assessed via multivariate logistic regression analysis.

RESULTS Patient demographics and comorbidities other than depression were similar in the 2 groups. In the Depression group, $85.1 \%$ of the patients were taking an antidepressant prior to surgery. There were no significant betweengroup differences in intraoperative variables and rates of complications other than delirium. Postoperative complication rates were also similar between the cohorts, including rates of urinary tract infection, fever, deep and superficial surgical site infection, pulmonary embolism, deep vein thrombosis, urinary retention, and proportion of patients transferred to the intensive care unit. In total, 66 patients $(7.15 \%)$ had an episode of postoperative delirium, with depressed patients experiencing approximately a 2 -fold higher rate of delirium (10.59\% vs $5.84 \%)$. In a multivariate logistic regression analysis, depression was an independent predictor of postoperative delirium after spine surgery in spinal deformity patients $(p=$ 0.01).

CONCLUSIONS The results of this study suggest that depression is an independent risk factor for postoperative delirium after elective spine surgery. Further studies are necessary to understand the effects of affective disorders on postoperative delirium, in hopes to better identify patients at risk.

https://thejns.org/doi/abs/10.3171/2017.4.SPINE161012

KEY WORDS depression; delirium; spine; deformity; surgery; outcomes

ABBREVIATIONS A-fib = atrial fibrillation; BMI = body mass index; CABG = coronary artery bypass graft; $C A D=$ coronary artery disease; $C H F=$ congestive heart failure; DVT = deep vein thrombosis; EBL = estimated blood loss; ICU = intensive care unit; $\mathrm{MI}=$ myocardial infarction; PE = pulmonary embolism; PRBC = packed red blood cell; PVD = peripheral vascular disease; SNRI = serotonin-norepinephrine reuptake inhibitor; SSI = surgical site infection; SSRI = selective serotonin reuptake inhibitor; UTI = urinary tract infection.

SUBMITTED August 30, 2016. ACCEPTED April 10, 2017.

INCLUDE WHEN CITING Published online June 2, 2017; DOI: 10.3171/2017.4.SPINE161012. 
$\mathrm{D}$ EPRESSION is the most common affective disorder in the United States, with $16.6 \%$ of the population experiencing at least 1 major depressive episode in their lifetime. ${ }^{8}$ Patients with spinal deformity in particular have been shown to be at an increased risk for depression when compared with the general population..$^{12,21}$ Spinal deformities can cause body image dissatisfaction, physical disability, and chronic pain, all of which may limit psychosocial functioning and increase the risk of depression. ${ }^{18}$ In addition, depression has been shown to be a significant risk factor for poor surgical outcomes after spine surgery, such as increased pain and postoperative disability. ${ }^{15,17}$

In particular, postoperative delirium has also been associated with increases in complication rates, hospital length of stay, in-hospital mortality, and overall health care costs., ${ }^{3,14}$ Due to the quality of care and economic implications of postoperative delirium, previous studies have attempted to determine independent associations and risk factors for postoperative delirium. In a retrospective study of 549 patients who underwent spinal surgery, Gao et al. ${ }^{4}$ found several preoperative risk factors for the development of postoperative delirium, including older age, number of medications, diabetes mellitus, and central nervous system disorders. However, the relationship between depression and postoperative delirium in spine surgery remains relatively unknown.

The aim of this study is to determine whether depression is an independent risk factor for postoperative delirium in spine deformity patients after spinal surgery.

\section{Methods}

In this retrospective analysis, the medical records of 923 adult (age $\geq 18$ years) spine deformity patients who had undergone elective spine surgery at Duke University Medical Center from 2005 through 2015 were reviewed. Institutional review board approval was obtained prior to study initiation. We included adult patients (age $\geq 18$ years) who met the following criteria: 1) diagnosis of spinal deformity, such as scoliosis and kyphosis, with radiographic evidence of degenerative scoliosis or spondylolisthesis; 2) undergoing elective corrective spinal surgery for spinal deformity; and 3) documented intra- and postoperative hospital course. Patients who had undergone spinal surgery previously or had any preexisting spinal pathology, including primary osseous pathology that might impair fusion, tumor, and infection, were excluded. We identified 255 patients (27.6\% of the overall group) who had a known history of depression and had received a diagnosis of depression from a board-certified psychiatrist prior to surgery (Depression cohort). The remaining 668 patients (72.4\%) made up the No-Depression cohort. The primary outcome of this study was the incidence of postoperative delirium.

Demographic variables evaluated included age, sex, and body mass index (BMI). Comorbidities included congestive heart failure (CHF), coronary artery disease (CAD), atrial fibrillation (A-fib), peripheral vascular disease (PVD), myocardial infarction (MI), hypertension, diabetes, deep vein thrombosis (DVT), hyperlipidemia, and pulmonary embolism (PE). Baseline alcohol use was recorded as well. For the Depression cohort, preoperative antidepressants were assessed and classified as selective serotonin reuptake inhibitors (SSRIs), serotonin-norepinephrine reuptake inhibitors (SNRIs), and/or other antidepressants, including tricyclic antidepressants, atypical antidepressants, and monoamine oxidase inhibitors.

Intraoperative variables and complications included number of vertebral levels involved, operative time, estimated blood loss (EBL), percent of patients requiring a blood transfusion, number of packed red blood cell (PRBC) transfusions, spinal cord injury, nerve root injury, and durotomy. Postoperative variables included length of hospital stay, intensive care unit (ICU) admission and length of stay, urinary tract infection (UTI), fever, ileus, deep and superficial surgical site infections (SSIs), hypertension, hypotension, hematoma, myocardial infarction (MI), pulmonary embolism (PE), deep vein thrombosis (DVT), sepsis, weakness, sensory deficit, urinary retention, and whether the patient was discharged with a Foley catheter. Data on postoperative neural deficits were also collected.

Parametric data were expressed as means (with standard deviations) and compared using the Student t-test. Nonparametric data were expressed as medians (with interquartile ranges) and compared via the Mann-Whitney U-test. Nominal data were compared with the chi-square test. The relationship between independent variables and postoperative delirium was assessed using a multivariate logistic regression model. All tests were 2 -sided, and results were considered statistically significant if the $p$ value was less than 0.05 . Statistical analysis was performed using JMP, Version 12 (SAS Institute Inc.).

\section{Results}

A total of 923 adult patients (age $\geq 18$ years) were included in this study-668 in the No-Depression cohort and 255 patients in the Depression cohort. There were no significant between-group differences in demographic characteristics, with similar proportions of men and similar mean age and BMI (Table 1). There were also no significant differences between the groups with respect to comorbidities, including CHF, CAD, A-fib, PVD, MI, hypertension, diabetes, DVT, hyperlipidemia, and PE, or alcohol consumption. In the Depression cohort, $85.1 \%$ of the patients were taking at least 1 antidepressant prior to surgery; $53.3 \%$ of the patients in this cohort were taking an SSRI, $22.0 \%$ were taking an SNRI, and 9.8\% were taking other types of antidepressants (Table 1).

The median number of vertebral levels involved in the procedure was similar in the No-Depression and Depression groups, as was the operative time, EBL, urine output (768.07 $\pm 737.52 \mathrm{ml}$ vs $680.94 \pm 595.84 \mathrm{ml}, \mathrm{p}=0.07)$, and number of PRBC transfusions (Table 2). The rate of incidental durotomy was similar in the 2 cohorts, and neither group had any direct intraoperative spinal cord or nerve root injuries.

\section{Postoperative Hospital Stay and Complication Profile}

Postoperative hospital stays were similar in the No- 
TABLE 1. Preoperative variables

\begin{tabular}{lccc}
\hline \multicolumn{1}{c}{ Variable } & $\begin{array}{c}\text { No Depression } \\
(\mathrm{n}=668)\end{array}$ & $\begin{array}{c}\text { Depression } \\
(\mathrm{n}=255)\end{array}$ & $\begin{array}{c}\mathrm{p} \\
\text { Value }\end{array}$ \\
\hline Preop variables & & & \\
\hline Male & $36.53 \%$ & $34.90 \%$ & 0.70 \\
\hline Age in yrs (mean) & $61.00 \pm 15.84$ & $61.87 \pm 11.59$ & 0.36 \\
\hline BMl in kg/m² (mean) & $28.39 \pm 6.84$ & $29.08 \pm 5.90$ & 0.14 \\
\hline CHF & $1.05 \%$ & $0.00 \%$ & 0.20 \\
\hline CAD & $11.08 \%$ & $10.20 \%$ & 0.81 \\
\hline A-fib & $5.69 \%$ & $4.71 \%$ & 0.63 \\
\hline PVD & $1.65 \%$ & $1.96 \%$ & 0.78 \\
\hline MI & $2.99 \%$ & $1.96 \%$ & 0.50 \\
\hline HTN & $55.24 \%$ & $61.57 \%$ & 0.09 \\
\hline DM & $14.82 \%$ & $13.33 \%$ & 0.60 \\
\hline DVT & $3.29 \%$ & $4.31 \%$ & 0.43 \\
\hline HLD & $37.28 \%$ & $38.82 \%$ & 0.70 \\
\hline PE & $1.80 \%$ & $3.53 \%$ & 0.14 \\
\hline Alcohol use & $29.64 \%$ & $29.02 \%$ & 0.87 \\
\hline Treatment of depression & & & \\
\hline Treated & & $85.1 \%$ & \\
\hline SSRI & & $53.3 \%$ & \\
\hline SNRI & & $22.0 \%$ & \\
\hline Other & & $9.8 \%$ & \\
\hline DM & & & \\
\hline
\end{tabular}

DM = diabetes mellitus; $\mathrm{HLD}=$ hyperlipidemia; $\mathrm{HTN}=$ hypertension.

Mean values are given with SDs.

Depression and Depression groups, including mean overall length of stay, ICU admission rates, and mean length of stay in the ICU for patients admitted to the ICU. The prevalence of postoperative complications was also similar in the 2 cohorts. Details are given in Table 3. A few patients had postoperative neurological deficits, but all of these deficits appeared to have resolved at 6 months after surgery.

There was a significant difference between the NoDepression and Depression groups with respect to postoperative delirium. In total, 66 patients $(7.15 \%)$ experienced an episode of postoperative delirium. Patients with depression had nearly a 2-fold higher incidence of postoperative delirium $(5.84 \%$ in the No-Depression group vs $10.59 \%$ in the Depression group, $\mathrm{p}=0.015$ ) (Table 3). When we examined types of delirious episodes in the 2 patient groups, we found no significant differences between groups with respect to the prevalence of hyperactive, hypoactive, or mixed delirious episodes. In the Depression group, among patients who experienced delirious episodes, $33.3 \%$ of episodes were hyperactive, $18.5 \%$ were hypoactive, and $33.3 \%$ were mixed delirious episodes; and in the No-Depression group, among patients who experienced delirious episodes, $23.1 \%$ of episodes were hyperactive, $10.3 \%$ were hypoactive, and $23.1 \%$ were mixed delirious episodes. Between-patient group comparisons of hyperactive, hypoactive, and mixed delirium episodes yielded $p=0.36, p=0.34$, and $p=0.36$, respectively. Furthermore, within the Depression cohort, there was no significant difference in the incidence of de-
TABLE 2. Intraoperative variables and complications

\begin{tabular}{lccc}
\hline \multicolumn{1}{c}{ Variable } & $\begin{array}{c}\text { No Depression } \\
(\mathrm{n}=668)\end{array}$ & $\begin{array}{c}\text { Depression } \\
(\mathrm{n}=255)\end{array}$ & $\begin{array}{c}\mathrm{p} \\
\text { Value }\end{array}$ \\
\hline No. of levels & & & \\
\hline Median & 4 & 4 & 0.61 \\
\hline IQR & $2-7$ & $2-6$ & \\
\hline $\begin{array}{c}\text { Operative time in } \\
\text { minutes (mean) }\end{array}$ & $274.33 \pm 138.67$ & $294.19 \pm 132.34$ & 0.054 \\
\hline EBL in ml (mean) & $798.90 \pm 1153.80$ & $959.33 \pm 1342.45$ & 0.09 \\
\hline $\begin{array}{c}\text { No. of PRBC trans- } \\
\text { fusions (mean) }\end{array}$ & $0.73 \pm 1.55$ & $0.89 \pm 1.59$ & 0.15 \\
\hline Spinal cord injury & $0 \%$ & $0 \%$ & 1.00 \\
\hline Nerve root injury & $0 \%$ & $0 \%$ & 1.00 \\
\hline Incidental durotomy & $5.84 \%$ & $7.84 \%$ & 0.29 \\
\hline
\end{tabular}

* 1 unit $=350 \mathrm{ml}$.

lirium between the patients who were receiving treatment for depression and those who were not $(11.1 \%$ vs $7.9 \%$, respectively, $\mathrm{p}=0.56$ ).

The complication profile was compared between patients who did and did not have delirium. There was a significant difference in the mean length of hospital stay $(8.4 \pm 3.9$ days for patients with delirium vs $5.1 \pm 3.7$ days for those without, $\mathrm{p}<0.001)$. Moreover, the proportion of patients transferred to the ICU was significantly greater in the group with delirium than in the group without delirium $(33.9 \%$ vs $18.1 \%, p=0.025)$, as was the percentage of patients with UTI $(19.7 \%$ vs $3.5 \%, \mathrm{p}<0.0001)$, ileus (12.1\% vs $4.7 \%, \mathrm{p}=0.017)$, and readmission within 30 days $(18.2 \%$ vs $9.7 \%, \mathrm{p}=0.036)$. All other complication rates were similar in the 2 cohorts.

\section{Postoperative Delirium Independent Predictors}

In a multivariate binary logistic regression analysis, depression $(p=0.01)$, patient age $(p<0.001)$, operative time $(\mathrm{p}=0.03)$, and postoperative UTI $(\mathrm{p}<0.001)$ were independent predictors of postoperative delirium (Table 4). Other patient variables, including sex, BMI, CHF, CAD, hypertension, PVD, diabetes mellitus, hyperlipidemia, and alcohol consumption, were not statistically significant independent predictors of postoperative delirium. Other operative variables and postoperative complications, including fusion levels, fever, deep SSI, superficial SSI, hypotension, UTI, and urinary retention, were also not statistically significant predictors.

\section{Discussion}

In this retrospective study of 923 adult patients (age $\geq$ 18 years) undergoing elective spinal surgery, depression emerged as an independent risk factor for the development of postoperative delirium.

Prior studies in various surgical fields have associated depression with an increased incidence of postoperative delirium. In a prospective study of 109 elderly patients (age $\geq 65$ years) undergoing surgery for acute hip fracture, Koskderelioglu et al.? demonstrated that depressive mood, 
TABLE 3. Postoperative complications

\begin{tabular}{lccc}
\hline \multicolumn{1}{c}{ Variable } & $\begin{array}{c}\text { No Depression } \\
(\mathrm{n}=668)\end{array}$ & $\begin{array}{c}\text { Depression } \\
(\mathrm{n}=255)\end{array}$ & $\begin{array}{c}\mathrm{p} \\
\text { Value }\end{array}$ \\
\hline LOS in days (mean) & $5.19 \pm 3.79$ & $5.70 \pm 3.89$ & 0.07 \\
\hline ICU & $20.36 \%$ & $16.46 \%$ & 0.21 \\
\hline $\begin{array}{l}\text { Length of ICU stay in } \\
\text { days (mean) }\end{array}$ & $3.09 \pm 19.33$ & $3.05 \pm 16.42$ & 0.98 \\
\hline Delirium & $5.84 \%$ & $10.59 \%$ & $0.015 *$ \\
\hline UTI & $4.04 \%$ & $6.27 \%$ & 0.16 \\
\hline Fever & $3.44 \%$ & $2.75 \%$ & 0.68 \\
\hline leus & $5.84 \%$ & $3.53 \%$ & 0.19 \\
\hline Deep SSI & $0.60 \%$ & $1.57 \%$ & 0.23 \\
\hline Superficial SSI & $0.75 \%$ & $1.18 \%$ & 0.69 \\
\hline HTN & $2.84 \%$ & $2.35 \%$ & 0.82 \\
\hline Hypotension & $5.34 \%$ & $7.06 \%$ & 0.34 \\
\hline Hematoma & $1.05 \%$ & $1.18 \%$ & 0.87 \\
\hline Ml & $0.45 \%$ & $1.18 \%$ & 0.36 \\
\hline PE & $0.75 \%$ & $1.57 \%$ & 0.27 \\
\hline DVT & $0.30 \%$ & $0.78 \%$ & 0.31 \\
\hline Sepsis & $1.66 \%$ & $0.39 \%$ & 0.20 \\
\hline Weakness & $5.09 \%$ & $5.88 \%$ & 0.62 \\
\hline Sensory deficit & $2.40 \%$ & $2.75 \%$ & 0.81 \\
\hline Urinary retention & $7.78 \%$ & $9.02 \%$ & 0.59 \\
\hline D/C w/ Foley catheter & $2.70 \%$ & $1.57 \%$ & 0.47 \\
\hline DI & & & \\
\hline
\end{tabular}

$\mathrm{D} / \mathrm{C}=$ discharge; $\mathrm{LOS}=$ length of stay.

* Statistically significant.

as assessed by the Beck Depression Inventory, correlated with the occurrence of postoperative delirium. In another prospective study of 485 elderly men undergoing transurethral prostatectomy for benign prostatic hyperplasia, Tai et al. ${ }^{16}$ showed that patients with a higher Geriatric Depression Scale (GDS) score were significantly more likely to develop postoperative delirium. Similarly, in a retrospective study of 224,060 patients undergoing elective total shoulder arthroplasty between 2002 and 2012, Mollon et al. ${ }^{10}$ showed depression to be an independent risk factor for postoperative delirium. In another retrospective analysis of 91 patients (age $\geq 75$ years) who underwent esophagectomy for the treatment of esophageal cancer, Yamamoto et al. ${ }^{20}$ showed that GDS score was independently associated with postoperative delirium. Analogous to these studies, our study demonstrated that adult patients with a diagnosis of depression prior to surgery were more likely to develop postoperative delirium than patients without a history of depression.

Previous studies have been conducted to elucidate the pathophysiology connecting depression and postoperative delirium. In a prospective cohort study of 113 patients undergoing coronary artery bypass graft (CABG) surgery, Kazmierski et al. ${ }^{5}$ found 2 factors that correlated with postoperative delirium: depression and elevated preoperative cortisol levels. However, once depression was controlled for, the association between cortisol levels and postoperative delirium lost its statistical significance. ${ }^{5}$ Therefore, this study hypothesized that depression is cor-
TABLE 4. Independent predictors of postoperative delirium

\begin{tabular}{lcc}
\hline \multicolumn{1}{c}{ Variable } & Coefficient & p Value \\
\hline Sex & 0.057 & 0.72 \\
\hline Age & 0.083 & $<0.0001^{*}$ \\
\hline Depression & 0.398 & $0.01^{*}$ \\
\hline BMI & 0.022 & 0.43 \\
\hline CHF & 0.385 & 0.56 \\
\hline CAD & 0.128 & 0.98 \\
\hline HTN & 0.049 & 0.64 \\
\hline PVD & 0.093 & 0.07 \\
\hline DM & -0.006 & 0.65 \\
\hline HLD & 0.084 & 0.95 \\
\hline Alcohol & 0.011 & 0.41 \\
\hline Fusion levels & -0.622 & 0.31 \\
\hline Operative time & -0.673 & $0.03^{*}$ \\
\hline Postop fever & 0.549 & 0.20 \\
\hline Postop deep SSI & 0.856 & 0.30 \\
\hline Postop superficial SSI & 0.320 & 0.44 \\
\hline Postop hypotension & 0.165 & 0.49 \\
\hline Postop UTI & 0.769 & $<0.001^{*}$ \\
\hline Postop urinary retention & 0.002 & 0.11 \\
\hline * Statistically significant $(p<0.05)$. & & \\
\hline
\end{tabular}

related with higher cortisol levels, and the elevated levels of stress hormones increase the risk of postoperative delirium. ${ }^{5}$ This conclusion is supported by other studies in the literature that have shown depression to be linked with increased cortisol levels, in both humans and nonhuman primates. ${ }^{13,19}$ Additionally, in a prospective study of 243 patients undergoing elective CABG surgery, Mu et al. ${ }^{11}$ found high cortisol levels to be linked with postoperative delirium. These elevated glucocorticoid levels have been hypothesized to cause structural changes in hippocampal neurons and to perturb the hypothalamic-pituitary axis, which may predispose the brain to episodes of delirium. ${ }^{6}$ In sum, this group of studies supports a potential link between depression, cortisol, and the development of postoperative delirium, lending a biological basis to this present research.

Postoperative delirium constitutes a significant financial burden on our health care system. In a study of $500 \mathrm{pa}-$ tients undergoing esophagectomy for malignancy, Markar et al. ${ }^{9}$ found that delirium was associated with a longer hospital stay (14 vs 10.9 days), longer ICU stay (3.6 vs 2.7 days), and increased cost ( $\$ 28,222.80$ vs $\$ 22,702.20)$. Similarly, in a retrospective study of 242 elderly patients (age $\geq 65$ years) who underwent surgical treatment for hip fracture in Canada, Zywiel et al. ${ }^{22}$ found that delirium increased the hospital stay following surgery by a mean length of 7.4 days and increased the cost of care by $\$ 8286$ (Canadian dollars). Furthermore, this study extrapolated that a $50 \%$ reduction in postoperative delirium rates could decrease health care costs by $\$ 50,708,000$ (Canadian dollars) each year in Canada, assuming that their results were generalizable across the entire country. ${ }^{22}$ These studies therefore suggest that utilization of preventive measures 
to reduce the incidence of postoperative delirium could significantly lessen the health care costs that have been soaring across the world.

Previous studies have identified possible preventive measures to improve postoperative outcomes in patients with depression. In a retrospective study of 140 adults who had undergone anterior cervical discectomy and fusion, Elsamadicy et al. ${ }^{2}$ found that patients with a history of depression that were pretreated with an antidepressant for at least 6 months prior to surgery had similar self-reported surgical outcomes to the control group that had no prior history of depression. Similarly, in a randomized control trial of 100 depressed patients who underwent CABG surgery, Dao et al. ${ }^{1}$ found that brief preoperative cognitive behavioral therapy reduced in-hospital length of stay (7.9 vs 9.2 days) in addition to improving depressive symptoms and quality of life. However, further studies are necessary to better understand the effects of depression on surgical outcomes.

The study has several limitations that may impact its interpretation. The variables were collected retrospectively and analyzed; therefore, this study is subject to the weaknesses of retrospective analysis, including reliance on record keeping of others, difficulty in determining temporal relationships, and selection and information bias. The chart review for this study involved only a single institution and may not reflect areas with different patient populations. Furthermore, data regarding the severity of the depressive symptoms and the duration of treatment were not collected for all patients and therefore these variables may have been subject to selection bias for the cohorts. Similarly, postoperative narcotic use and complete metabolic panels were not collected and assessed in all patients, which may have implications on our analysis (not accounting for potentially confounding factors and isolated metabolic differences between the cohorts). Despite these limitations, this study has shown that depression is an independent risk factor for the development of postoperative delirium in our patient population.

\section{Conclusions}

The results of this study suggest that depression is an independent risk factor for postoperative delirium after elective spine surgery. Further studies are necessary to understand the effects of affective disorders on postoperative delirium, in the hope of better identifying at-risk patients and improving patient care.

\section{References}

1. Dao TK, Youssef NA, Armsworth M, Wear E, Papathopoulos KN, Gopaldas R: Randomized controlled trial of brief cognitive behavioral intervention for depression and anxiety symptoms preoperatively in patients undergoing coronary artery bypass graft surgery. J Thorac Cardiovasc Surg 142:e109-e115, 2011

2. Elsamadicy AA, Adogwa O, Cheng J, Bagley C: Pretreatment of depression before cervical spine surgery improves patients' perception of postoperative health status: a retrospective, single institutional experience. World Neurosurg 87:214-219, 2016

3. Franco K, Litaker D, Locala J, Bronson D: The cost of delirium in the surgical patient. Psychosomatics 42:68-73, 2001

4. Gao R, Yang ZZ, Li M, Shi ZC, Fu Q: Probable risk factors for postoperative delirium in patients undergoing spinal surgery. Eur Spine J 17:1531-1537, 2008

5. Kazmierski J, Banys A, Latek J, Bourke J, Jaszewski R: Cortisol levels and neuropsychiatric diagnosis as markers of postoperative delirium: a prospective cohort study. Crit Care 17:R38, 2013

6. Kazmierski J, Kloszewska I: Is cortisol the key to the pathogenesis of delirium after coronary artery bypass graft surgery? Crit Care 15:102, 2011

7. Koskderelioglu A, Onder O, Gucuyener M, Altay T, Kayali C, Gedizlioglu M: Screening for postoperative delirium in patients with acute hip fracture: assessment of predictive factors. Geriatr Gerontol Int [epub ahead of print], 2016

8. Kessler RC, Petukhova M, Sampson NA, Zaslavsky AM, Wittchen HU: Twelve-month and lifetime prevalence and lifetime morbid risk of anxiety and mood disorders in the United States. Int J Methods Psychiatr Res 21:169-184, 2012

9. Markar SR, Smith IA, Karthikesalingam A, Low DE: The clinical and economic costs of delirium after surgical resection for esophageal malignancy. Ann Surg 258:77-81, 2013

10. Mollon B, Mahure SA, Ding DY, Zuckerman JD, Kwon YW: The influence of a history of clinical depression on perioperative outcomes in elective total shoulder arthroplasty: a ten-year national analysis. Bone Joint J 98-B:818-824, 2016

11. Mu DL, Wang DX, Li LH, Shan GJ, Li J, Yu QJ, et al: High serum cortisol level is associated with increased risk of delirium after coronary artery bypass graft surgery: a prospective cohort study. Crit Care 14:R238, 2010

12. Payne WK III, Ogilvie JW, Resnick MD, Kane RL, Transfeldt EE, Blum RW: Does scoliosis have a psychological impact and does gender make a difference? Spine (Phila Pa 1976) 22:1380-1384, 1997

13. Qin DD, Rizak J, Feng XL, Yang SC, Lü LB, Pan L, et al: Prolonged secretion of cortisol as a possible mechanism underlying stress and depressive behaviour. Sci Rep 6:30187, 2016

14. Rudolph JL, Marcantonio ER: Review articles: postoperative delirium: acute change with long-term implications. Anesth Analg 112:1202-1211, 2011

15. Sinikallio S, Aalto T, Airaksinen O, Herno A, Kröger H, Savolainen S, et al: Depression is associated with poorer outcome of lumbar spinal stenosis surgery. Eur Spine J 16:905-912, 2007

16. Tai S, Xu L, Zhang L, Fan S, Liang C: Preoperative risk factors of postoperative delirium after transurethral prostatectomy for benign prostatic hyperplasia. Int J Clin Exp Med 8:4569-4574, 2015

17. Tetreault L, Nagoshi N, Nakashima H, Singh A, Kopjar $\mathrm{B}$, Arnold P, et al: The impact of depression and bipolar disorders on functional and quality of life outcomes in patients undergoing surgery for degenerative cervical myelopathy: analysis of a combined prospective dataset. Spine (Phila Pa 1976) 42:372-378, 2017

18. Tones M, Moss N, Polly DW Jr: A review of quality of life and psychosocial issues in scoliosis. Spine (Phila Pa 1976) 31:3027-3038, 2006

19. Verduijn J, Milaneschi Y, Schoevers RA, van Hemert AM, Beekman AT, Penninx BW: Pathophysiology of major depressive disorder: mechanisms involved in etiology are not associated with clinical progression. Transl Psychiatry 5:e649, 2015

20. Yamamoto M, Yamasaki M, Sugimoto K, Maekawa Y, Miyazaki Y, Makino T, et al: Risk evaluation of postoperative delirium using comprehensive geriatric assessment in elderly patients with esophageal cancer. World J Surg 40:2705-2712, 2016 
21. Zhang J, He D, Gao J, Yu X, Sun H, Chen Z, et al: Changes in life satisfaction and self-esteem in patients with adolescent idiopathic scoliosis with and without surgical intervention. Spine (Phila Pa 1976) 36:741-745, 2011

22. Zywiel MG, Hurley RT, Perruccio AV, Hancock-Howard RL, Coyte PC, Rampersaud YR: Health economic implications of perioperative delirium in older patients after surgery for a fragility hip fracture. J Bone Joint Surg Am 97:829-836, 2015

\section{Disclosures}

Dr. Karikari reports a consultant relationship with NuVasive.

\section{Author Contributions}

Conception and design: Adogwa, Elsamadicy. Acquisition of data: Elsamadicy, Lydon, Sergesketter, Kaakati. Analysis and interpretation of data: Adogwa, Elsamadicy. Drafting the article: Adogwa, Elsamadicy, Lydon, Sergesketter, Kaakati. Critically revising the article: all authors. Reviewed submitted version of manuscript: all authors. Approved the final version of the manuscript on behalf of all authors: Adogwa. Statistical analysis: Adogwa, Elsamadicy. Administrative/technical/material support: Mehta, Vasquez, Cheng, Bagley, Karikari. Study supervision: Mehta, Vasquez, Cheng, Bagley, Karikari.

\section{Correspondence}

Owoicho Adogwa, Department of Neurosurgery, Rush University Medical Center, 1725 W Harrison, Ste. 855, Chicago, IL 60612. email: owoicho.adogwa@gmail.com. 